Acta Crystallographica Section D

Biological

Crystallography

ISSN 0907-4449

Yanhui Xu, ${ }^{a, b}$ Zhihong Bai, ${ }^{a, b}$ Lan Qin, ${ }^{a, b} \mathrm{Xu} \mathrm{Li,}{ }^{\mathrm{a}, \mathrm{b}}$ George $\mathrm{Gao}^{\mathrm{c}}$ and Zihe $\mathbf{R a o}^{\mathbf{a}, \mathbf{b}_{*}}$

aLaboratory of Structural Biology, Tsinghua University, Beijing 100084, People's Republic of China, ${ }^{\mathbf{b}}$ National Laboratory of Biomacromolecules, Institute of Biophysics, Beijing 100101, People's Republic of China, and ${ }^{\mathbf{C}}$ Nuffield Department of Clinical Medicine, John Radcliffe Hospital, Oxford University, Oxford OX3 9DU, England

Correspondence e-mail: raozh@xtal.tsinghua.edu.cn

\section{Crystallization and preliminary crystallographic analysis of the fusion core of the spike protein of the murine coronavirus mouse hepatitis virus (MHV)}

Crystals of a 2-Helix fusion-core construct of MHV spike protein

Received 25 April 2004 (commonly referred to as E2) have been grown at $291 \mathrm{~K}$ using PEG Accepted 17 August 2004
4000 as precipitant. The diffraction pattern of the crystal extends to $2.8 \AA$ resolution at $100 \mathrm{~K}$ in-house. Furthermore, a selenomethionine (SeMet) derivative of MHV spike protein fusion core has been overexpressed and purified. The derivative crystals were obtained under similar conditions and three different wavelength data sets were collected to $2.4 \AA$ resolution from a single derivative crystal at BSRF (Beijing Synchrotron Radiation Facility). The crystals have unit-cell parameters $a=b=48.3, c=199.6 \AA$, $\alpha=\beta=90, \gamma=120^{\circ}$ and belong to space group R3. Assuming the presence of two molecules in the asymmetric unit, the solvent content is calculated to be about $46 \%$.

\section{Introduction}

Mouse hepatitis virus strain A59 (MHV-A59) belongs to the coronaviruses, which comprise a large and diverse family of enveloped viruses with a single-stranded positive-sense RNA genome of approximately $31000 \mathrm{bp}$ (Lee et al., 1991; Spaan et al., 1988). The Coronaviridae exhibit a broad host range, infecting many mammalian and avian species and causing upper respiratory, gastrointestinal, hepatic and central nervous system diseases. In humans and birds coronaviruses primarily cause upper respiratory tract infections, while porcine and bovine coronaviruses establish enteric infections that result in severe economic loss (Siddell, 1995).

The coronavirus spike (S) protein, which is also commonly referred to as E2, forms the peplomer structure on the viral envelope and each spike is thought to be a dimer or trimer (Cavanagh, 1983). The $\mathrm{S}$ protein mediates binding of virions to the host-cell receptor (Collins et al., 1982), viral fusion during entry and cell-to-cell fusion at the later stage of postinfection (Vennema et al., 1990). The MHV S protein is cleaved in the Golgi apparatus by a host-cell protease into two similarly sized subunits: the amino-terminal S1 and the carboxyl-terminal S2 (Frana et al., 1985; Luytjes et al., 1987; Sturman et al., 1985). It is believed that the $\mathrm{S} 1$ subunit forms the globular head of the spike, whereas the S2 subunit forms the transmembrane stalk portion (de Groot et al., 1987). Sequence analysis suggests that the coronavirus spike protein has the structural features of a type I membrane protein, including two heptad-repeat regions, a fusion peptide, a transmembrane domain near the carboxyl-terminus of S2 and a hydrophobic signal peptide at the N-terminus of S1 (Spaan et al., 1988). The S2 domain has an internal fusion peptide and two heptad-repeat regions, designated HR1 and HR2, respectively. HR2 is located close to the transmembrane region and HR1 is some 170 amino acids upstream of it.

Typical class I viral fusion proteins contain several notable features. They all have a fusion peptide at the amino-terminus that is thought to insert directly into the target membrane during the viral fusion process. Carboxylterminal to the fusion peptide are two regions containing 4,3 hydrophobic (heptad) repeats, sequence motifs that form a coiled-coil structure. This structure forms a stable protease K-resistant core in the ectodomain of the enveloped glycoprotein (or fusion protein). The fusion-core complex consists of two peptides, termed HR1 and HR2 peptides, that correspond to the two regions with hydrophobic heptad repeats. Heptad-repeat (HR) regions are found in the fusion proteins of many different viruses and form an important characteristic of class I viral fusion proteins (Bosch et al., 2003; Eckert \& Kim, 2001). Previous biophysical and structural studies show that the HR1 and HR2 peptides of MHV spike protein form a stable helical trimer of heterodimers (Bosch et al., 2003). It is believed that the two HR domains refold into a six-helix bundle during the fusion process, in which the HR1 domain forms a trimeric coiled coil surrounded by three antiparallel helices of the HR2 domain (Eckert \& Kim, 2001). Consequently, the transmembrane domain and the fusion peptide, which is known to insert into
(C) 2004 International Union of Crystallography Printed in Denmark - all rights reserved 
the cell membrane, are both transposed into close association. This pulls the cellular and viral membranes into proximity, facilitating the membrane fusion.

Our recent experiments have shown that the two HR domains of MHV spike protein can also form a six-helix bundle (Xu et al., in preparation). Here, we report the preliminary X-ray crystallographic analysis of the first fusion-core complex of a coronavirus spike protein, namely the MHV fusion core.

\section{Materials and methods}

\subsection{Expression and purification of the fusion-core proteins}

The fusion-core proteins of MHV were prepared as a single chain by linking the HR1 and HR2 domains via an eight-aminoacid linker (GGSGGSGG). The amino-acid sequences of HR1 and HR2 were taken from the murine coronavirus mouse hepatitis virus strain A59 (GeneBank No. M18379). The HR1 region used was derived from amino acids 968-1027 and HR2 from 1216-1254. The constructs and the encoded proteins were called 2-Helix (Fig. 1). The preparation and characterization of the 2-Helix proteins will be reported elsewhere (Xu et al., in preparation).

The construct was cloned into the NdeI and XhoI sites (introduced by synthetic PCR primers) of pET expression vector pET22b (Novagen). The construct was verified by sequencing, the expected plasmid was transformed into Escherichia coli strain BL21(DE3) competent cells and the transformants were selected on LB agar plates containing $100 \mu \mathrm{g} \mathrm{ml}^{-1}$ ampicillin. The cells were cultured at $310 \mathrm{~K}$ in $2 \times \mathrm{YT}$ medium containing $100 \mu \mathrm{g} \mathrm{ml}^{-1}$ ampicillin. When the culture density $\left(A_{600}\right)$ reached $0.6-0.8$, the culture was induced with $0.2 \mathrm{~m} M$ IPTG and grown for an additional $10 \mathrm{~h}$ at $289 \mathrm{~K}$ before the cells were harvested.

The bacterial cell pellet was resuspended in PBS and homogenized by sonication. The lysate was centrifuged at $18000 \mathrm{~g}$ for $20 \mathrm{~min}$ at $277 \mathrm{~K}$ and the supernatant was loaded onto an $\mathrm{Ni}^{2+}$-NTA column (Qiagen). The contaminated protein was washed with washing buffer $(1 \times \mathrm{PBS}, 60 \mathrm{~m} M$ imidazole $)$ and the target protein was eluted with elution buffer $(1 \times$ PBS, $500 \mathrm{~m} M$ imidazole $)$. The protein purified by affinity chromatography was further purified using a Superdex 75 column (Pharmacia) and analyzed by SDS-PAGE.

The selenomethionine derivative of the MHV 2-Helix was expressed using E. coli BL21(DE3) cultured in minimal medium M9 containing $60 \mathrm{mg} \mathrm{l}^{-1}$ L-SetMet. Six amino acids (lysine, threonine, phenylalanine, leucine, isoleucine and valine) were added to the culture for inhibited Met biosynthesis of the BL21(DE3) expression strain. Purification of the selenomethionene MHV 2-Helix was performed as for the native MHV 2-Helix. The incorporation of selenium was confirmed by mass-spectrometric analysis.

\subsection{Crystallization}

The purified protein was dialyzed against crystallization buffer $(10 \mathrm{~m} M$ Tris- $\mathrm{HCl} \mathrm{pH}$ $8.0,10 \mathrm{mM} \mathrm{NaCl}$ ) and concentrated to 8$10 \mathrm{mg} \mathrm{ml}^{-1}$. Protein concentrations were determined by absorbance at $280 \mathrm{~nm}$, assuming an $A_{280}$ of 0.22 for a $1.0 \mathrm{mg} \mathrm{ml}^{-1}$ solution. Initial crystallization conditions were screened using Crystal Screen reagent kits (Hampton Research). The protein could be crystallized under several conditions. Conditions yielding crystals were further optimized by variation of precipitant and protein concentration and additives. Goodquality crystals could be obtained in $0.1 \mathrm{M}$ MES pH 6.5, 10\%(v/v) PEG 4000, 8\%(v/v)

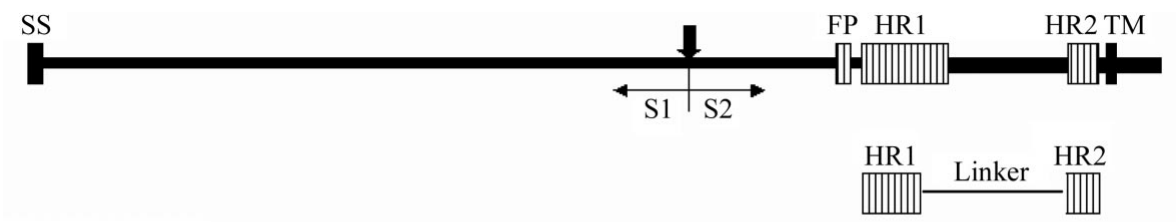

MHV 2-Helix MENQKMIASAFNNALGAIQDGFDATNSALGKIQSVVNANAEALNNLLNQLSNRFGAISASLGGSGGSGG DLSLDFEKLNVTLLDLTYEMNRIQDAIKKLNESYINLKE

Figure 1

Schematic representation of coronavirus MHV A59 spike protein and the MHV 2-Helix constructs. S1 and S2 are formed after proteolytic cleavage (vertical arrow) and noncovalently linked. The enveloped protein has an $\mathrm{N}$-terminal signal sequence (SS) and a transmembrane domain (TM) adjacent to the C-terminus. S2 contains two HR (heptad-repeat) regions (hatched bars), termed HR1 and HR2. FP (hatched bars) is a putative fusion peptide followed by an HR1 region. Two HR regions were linked to a single polypeptide with an eight-residue linker (GGSGGSGG).

DMSO and $5 \mathrm{~m} M$ hexaminecobalt trichloride (Figs. $2 a$ and $2 b$ ). Crystallization was performed by the hanging-drop vapourdiffusion method at $291 \mathrm{~K} .1 \mu \mathrm{l}$ protein solution was mixed with $1 \mu \mathrm{l}$ reservoir solution and the mixture was equilibrated against $200 \mu \mathrm{l}$ reservoir solution at $291 \mathrm{~K}$. The crystals appeared in $3 \mathrm{~d}$.

The purified selenomethionine derivative of MHV 2-Helix was concentrated to $8 \mathrm{mg} \mathrm{ml}^{-1}$. Crystallization trials were set up based on the optimum conditions used for native protein.

\subsection{Data collection and processing}

Crystals were soaked for a few seconds in a solution containing $50 \%(w / v)$ polyvinylpyrrolidone $\mathrm{K} 59$, which served as a cryoprotectant. The crystal was mounted in nylon loops and flashed-cooled in a cold nitrogen-gas stream at $100 \mathrm{~K}$ using an Oxford Cryostream. Data collection was performed by the rotation method using a MAR CCD detector with synchrotron radiation at BSRF (beamline $3 \mathrm{~W} 1 \mathrm{~A}$ of

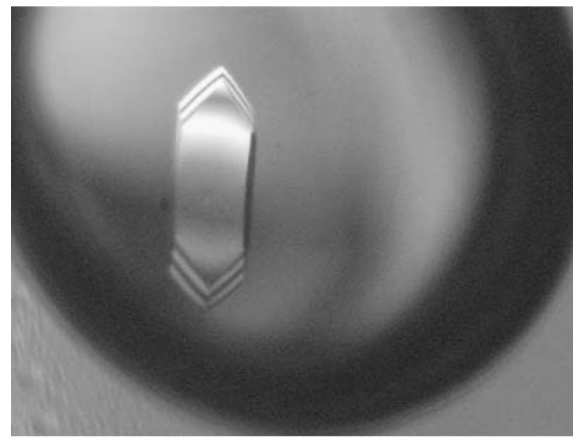

(a)

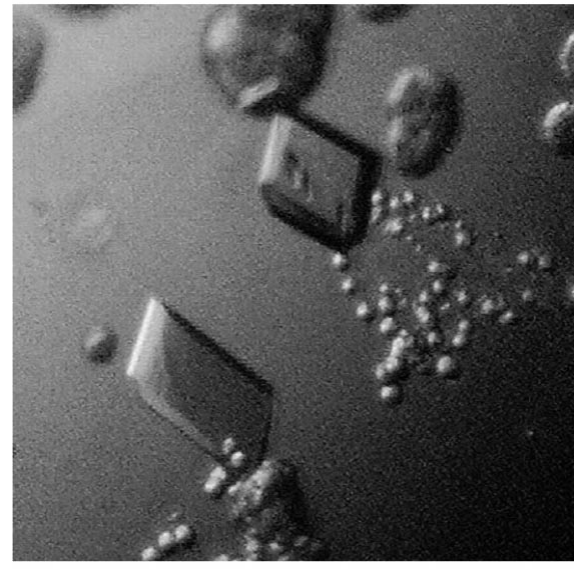

(b)

Figure 2

(a) Typical crystals of the MHV 2-Helix proteins grown using the hanging-drop method in $0.1 \mathrm{M}$ MES $\mathrm{pH} 6.5,6 \%(v / v)$ PEG 4000. (b) Typical crystals of the MHV 2-Helix protein (selenomethionine derivative) grown using the hanging-drop method in 0.1 $\mathrm{M}$ MES $\mathrm{pH} 6.5,10 \%(v / v)$ PEG 4000, 8\%(v/v) DMSO and $5 \mathrm{~m} M$ hexaminecobalt trichloride. 
Table 1

Data-collection and processing statistics.

Values in parentheses correspond to the highest resolution shell.

\begin{tabular}{lllll}
\hline Data & Peak & Edge & Remote & Native \\
\hline Space group & $R 3$ & $R 3$ & $R 3$ & $R 3$ \\
Unit-cell parameters $(\AA)$ & & & 48.3 & 48.3 \\
$\quad a(\AA)$ & 48.4 & 48.5 & 48.3 & 48.3 \\
$\quad b(\AA)$ & 48.4 & 48.5 & 199.4 & 199.6 \\
$\quad c(\AA)$ & 200.0 & 200.1 & 0.9000 & 0.9000 \\
Wavelength $(\AA)$ & 0.9799 & 0.9801 & $35-2.4$ & $35-2.5$ \\
Resolution range $(\AA)$ & $35-2.4$ & $35-2.4$ & 49352 & 43687 \\
Observed reflections & 49717 & 50167 & 6804 & 5901 \\
Unique reflections & 6842 & 6867 & $100(100)$ & $100(100)$ \\
Completeness $(\%)$ & $100(100)$ & $100(100)$ & $13.7(3.8)$ & $12.4(5.6)$ \\
$\langle I / \sigma(I)\rangle$ & $14.3(4.1)$ & $14.6(4.5)$ & $9.3(36.1)$ & $7.4(24.2)$ \\
$R_{\text {merge }}(\%)$ & $9.4(34.0)$ & $8.4(31.0)$ & &
\end{tabular}

$\dagger R_{\text {merge }}=\sum_{h} \sum_{i}\left|I_{h, i}-I_{h}\right| / \sum_{h} \sum_{i} I_{h, i}$ for the intensity $(I)$ of $i$ observations of reflection $h$. The native data were reprocessed using remote data in non-anomalous form with the resolution range $35-2.5 \AA$.

Beijing Synchrotron Radiation Facility). Data were indexed, integrated and scaled using DENZO and SCALEPACK from the $H K L$ program suite (Otwinowski \& Minor, 1997).

\section{Results and discussion}

The MHV 2-Helix protein construct could be crystallized under several conditions. Large birefringent parallelopiped crystals could be obtained using 0.1 $M$ MES pH 6.5, $6 \%(v / v)$ PEG 4000, although the diffraction pattern of the crystals only extended to $8 \AA$ resolution (Fig. 2a). Well diffracting crystals could only be obtained using $0.1 M \mathrm{MES} \mathrm{pH}$ $6.5,10 \%(v / v)$ PEG 4000, 8\%(v/v) DMSO and $5 \mathrm{~m} M$ hexaminecobalt trichloride (Fig. $2 b$ ). The crystals belong to space group $R 3$, with unit-cell parameters $a=b=48.3$, $c=199.6 \AA, \alpha=\beta=90, \gamma=120^{\circ}$. Assuming the presence of two molecules in the asym- metric unit, the solvent content is calculated to be about $46 \%$. Selected data statistics are shown in Table 1.

Multiple-wavelength anomalous dispersion (MAD) data were collected from a single selenomethionine-derivative crystal at peak (0.9799 $\AA$ ), inflection (0.9801 $\mathrm{\AA})$ and remote $(0.900 \AA)$ wavelengths to $2.4 \AA$. The structure of MHV 2-Helix has been determined by the MAD phasing method and will be published elsewhere.

Clear structural analysis of the fusion core of MHV would provide a detailed picture of the viral fusion-core structure (the first crystal structure of the fusion core of a coronavirus spike protein) and the viral fusion mechanisms mediated by the spike protein of coronaviruses. This will add to the repertoire of viral fusion-core structures. This will also open a new avenue towards the structure-based fusion-inhibitor design of peptides or peptide analogues, e.g. small molecules, for these emerging infectious diseases.

This work was supported by Projects 973 and 863 of the Ministry of Science and Technology of China (Grant Nos. 200BA711A12, G199075600 and 2003CB514116) and The Key Project of the Knowledge Innovation Program of Chinese Academy of Sciences (CAS). GFG's stay at Tsinghua University was supported by the Chunhui Project Scheme of the Ministry of Education of China.

\section{References}

Bosch, B. J., van der Zee, R., de Haan, C. A. \& Rottier, P. J. (2003). J. Virol. 77, 8801-8811.

Cavanagh, D. (1983). J. Gen. Virol. 64, 2577-2583.

Collins, A. R., Knobler, R. L., Powell, H. \& Buchmeier, M. J. (1982). Virology, 119, 358-371.

Eckert, D. M. \& Kim, P. S. (2001). Annu. Rev. Biochem. 70, 777-810.

Frana, M. F., Behnke, J. N., Sturman, L. S. \& Holmes, K. V. (1985). J. Virol. 56, 912-920.

Groot, R. J. de, Luytjes, W., Horzinek, M. C., van der Zeijst, B. A., Spaan, W. J. \& Lenstra, J. A. (1987). J. Mol. Biol. 196, 963-966.

Lee, H. J., Shieh, C. K., Gorbalenya, A. E., Koonin, E. V., La Monica, N., Tuler, J., Bagdzhadzhyan, A. \& Lai, M. M. (1991). Virology, 180, 567-582.

Luytjes, W., Sturman, L. S., Bredenbeek, P. J., Charite, J., van der Zeijst, B. A., Horzinek, M. C. \& Spaan, W. J. (1987). Virology, 161, 479-487.

Otwinowski, Z. \& Minor, W. (1997). Methods Enzymol. 276, 307-326.

Siddell, S. G. (1995). The Coronaviridae: an Introduction, pp. 1-10. New York: Plenum.

Spaan, W., Cavanagh, D. \& Horzinek, M. C. (1988). J. Gen. Virol. 69, 2939-2952.

Sturman, L. S., Ricard, C. S. \& Holmes, K. V. (1985). J. Virol. 56, 904-911.

Vennema, H., Heijnen, L., Zijderveld, A., Horzinek, M. C. \& Spaan, W. J. (1990). J. Virol. 64, 339-346. 